\title{
BMJ Open Bee venom acupuncture for rheumatoid arthritis: a systematic review protocol
}

\author{
Ju Ah Lee, Mi Ju Son, Jiae Choi, Kyung-Jin Yun, Ji Hee Jun, Myeong Soo Lee
}

To cite: Lee JA, Son MJ, Choi J, et al. Bee venom acupuncture for rheumatoid arthritis: a systematic review protocol. BMJ Open 2014;4: e004602. doi:10.1136/ bmjopen-2013-004602

- Prepublication history and additional material for this paper is available. To view please visit the journal (http://dx.doi.org/10.1136/ bmjopen-2013-004602).

Received 3 December 2013 Revised 15 March 2014 Accepted 18 March 2014

Korea Institute of Oriental Medicine, Daejeon, South Korea

Correspondence to Dr Myeong Soo Lee; drmslee@gmail.com

\section{ABSTRACT}

Introduction: This systematic review aims to analyse the trial data on the effects of bee venom acupuncture (BVA) for rheumatoid arthritis (RA).

Methods and analysis: The following 14 databases will be searched from their inception to March 2014: MEDLINE, EMBASE, Cochrane Central Register of Controlled Trials (CENTRAL), AMED, CINAHL, six Korean medical databases (OASIS, Korean Traditional Knowledge Portal, Korean Studies Information Service System, KoreaMed, Korean Medical Database and DBPIA) and three Chinese databases including CNKI (China National Knowledge Infrastructure), Wanfang and VIP. The methodological quality will be assessed using the Cochrane risk of bias tool.

Dissemination: The systematic review will be published in a peer-reviewed journal. The review will also be disseminated electronically and in print.

Trial registration number: PROSPERO 2013: CRD42013005853

\section{INTRODUCTION}

\section{Description of the condition}

Rheumatoid arthritis (RA) is a chronic inflammatory autoimmune disorder that results in pain and stiffness, joint swelling, deformity of joints and ankylosis develop. The complex, systemic nature of the disease makes RA treatment complex and involving a variety of approaches. The major aims of treatment are to relieve pain and swelling, reduce inflammation and joint damage, prevent disability and preserve or improve patients' well-being and function. ${ }^{1}$

Untreated RA leads to joint destruction, functional limitation and severe disability ${ }^{2}{ }^{3}$ and has a significant impact on healthrelated quality of life (HRQoL). ${ }^{4}$

\section{Description of the intervention}

Bee venom (BV) therapy has been used since ancient times, including the administration of honeybee stings, BV injection and BV acupuncture (BVA). ${ }^{6}$ BVA involves injecting purified, diluted bee venom into acupoints or ashi-points on the body. ${ }^{7}$

\section{Strengths and limitations of this study}

- The strength of this systematic review is its extensive, unbiased search of various databases without a language restriction.

- The trial screening and data extraction will be conducted independently by two authors.

- A possible weakness may be the quality of the trials that we identify because the complementary and alternative medicine research field has not been explored deeply.

How the intervention might work

BVA exhibits several pharmacological actions, including analgesic, anti-inflammatory, antiarthritic and anticancer effects through multiple mechanisms, such as the activation of the central inhibitory and excitatory systems and modulation of the immune system. ${ }^{8}$ The analgesic effects of BVA have been reported in animal experiments ${ }^{9} 10$ and in the clinic. ${ }^{711}$ In many countries, including the USA, BV therapy has been used to treat multiple sclerosis and arthritis. ${ }^{12}{ }^{13}$ However, most of these therapeutic uses are not based on evidence.

Why it is important to perform this review Currently, BVA for RA is widely used as an effective method. However, there is no critically appraised evidence, such as a systematic review or meta-analysis, of the potential benefits and harm of BVA for RA. A comprehensive evaluation of the effects of BVA for RA will help manage patients using BVA treatment.

\section{Objectives}

We will perform a systematic review to assess the effects of BVA for treating RA.

\section{METHODS}

Criteria for including studies in this review Types of studies

All prospective randomised controlled clinical trials (RCTs) and quasi-RCTs will be included. 
Types of participants

Patients suffering from RA will be included.

\section{Types of interventions}

We will include RCTs of BV injection at acupoints or ashi-points on the body as the sole treatment or as an adjunct to other treatments if the control group received the same treatment as the BVA group. Trials comparing BVA with any type of control intervention will be included. We will exclude trials of BV injection into parts of the body or ashi-points. Trials will also be excluded if only immunological or biological parameters were assessed. We will also exclude trials comparing two different types of BVA.

\section{Types of outcome measures}

\section{Primary outcomes}

Symptom (morning stiffness, pain and joint swelling) evaluation.

\section{Secondary outcomes}

- The number of joints affected by RA

- Adverse effects likely to be related to RA

- Quality of life

- Erythrocyte sedimentation rate (ESR)

- $\mathrm{C}$ reactive protein $(\mathrm{CRP})$

- Rheumatoid factor (RF)

\section{Search methods for identifying the studies}

\section{Electronic searches}

We will search for trials in the following electronic databases from their inception to March 2014: MEDLINE, EMBASE, Cochrane Central Register of Controlled Trials (CENTRAL), AMED and CINAHL. We will also search six Korean medical databases (OASIS, Korean Traditional Knowledge Portal, Korean Studies Information Service System, KoreaMed, Korean Medical Database and DBPIA) and three Chinese databases including CNKI (China National Knowledge Infrastructure), Wanfang and VIP.

\section{Searching other resources}

We will also perform non-electronic searches of conference proceedings, our own files of articles and nine Korean traditional medical journals (Journal of Korean Medicine, The Journal of Korean Acupuncture and Moxibustion Society, Korean Journal of Acupuncture, Journal of Acupuncture and Meridian Studies, Journal of Pharmacopuncture, Journal of Oriental Rehabilitation Medicine, The Journal of Korea Chuna Manual Medicine for Spine and Nerves, Korean Journal of Oriental Physiology and Pathology, and The Journal of Korean Oriental Internal Medicine).

\section{Search strategy}

The strategy for searching the MEDLINE database is presented in online supplementary appendix 1 . Similar search strategies will be applied for the other databases.
Data collection and analysis

Study selection

The data screening and selection process will be performed independently by four authors and will be verified by the fifth author $(\mathrm{JHJ})$, who is fluent in Chinese. When disagreements on the selection are not resolved through discussions, the arbiter (MSL) will decide. No language restrictions will be imposed. Hard copies of all articles will be obtained and read in full. Details of the selection process will be shown in the PRISMA flow diagram (figure 1).

\section{Data extraction and management}

The data extraction and quality assessment will be conducted by three authors (JAL, MJS and JHJ) using a predefined data extraction form.

Any disagreement among the authors will be resolved by discussion with all of the authors. When the reported data are insufficient or ambiguous, MSL will contact the corresponding authors by e-mail or telephone to request additional information or clarification.

\section{Assessment of bias in the included studies}

We will independently assess bias in the included studies according to the criteria from the Cochrane Handbook V.5.1.0, which include random sequence generation, allocation concealment, blinding of participants and personnel, blinding of outcome assessment, incomplete outcome data, selective reporting and other sources of bias. ${ }^{14}$ The quality of each trial will be categorised into a low, unclear or high risk of bias. If necessary, we will contact the authors of the assessed trials for clarification. We will resolve any differences in opinion through discussion or consultation with a third author.

\section{Measurement of the treatment effect}

For continuous data, we will use the mean difference (MD) with 95\% CIs to measure the treatment effect. We will convert other forms of data into MDs. In the case of outcome variables with different scales, we will use the standard mean difference with $95 \%$ CIs. For dichotomous data, we will present the treatment effect as a relative risk (RR) with 95\% CIs. We will convert other binary data into an RR value.

\section{Unit of analysis issues}

For cross-over trials, data from the first treatment period will be used. For trials in which more than one control group was assessed, the primary analysis will combine the data from each control group. Subgroup analyses of the control groups will also be performed. Each patient will be counted only once in the analysis.

\section{Dealing with the missing data}

We will contact the original authors for missing data whenever possible. If it is not possible to get the missing data, we will only analyse the available data. 


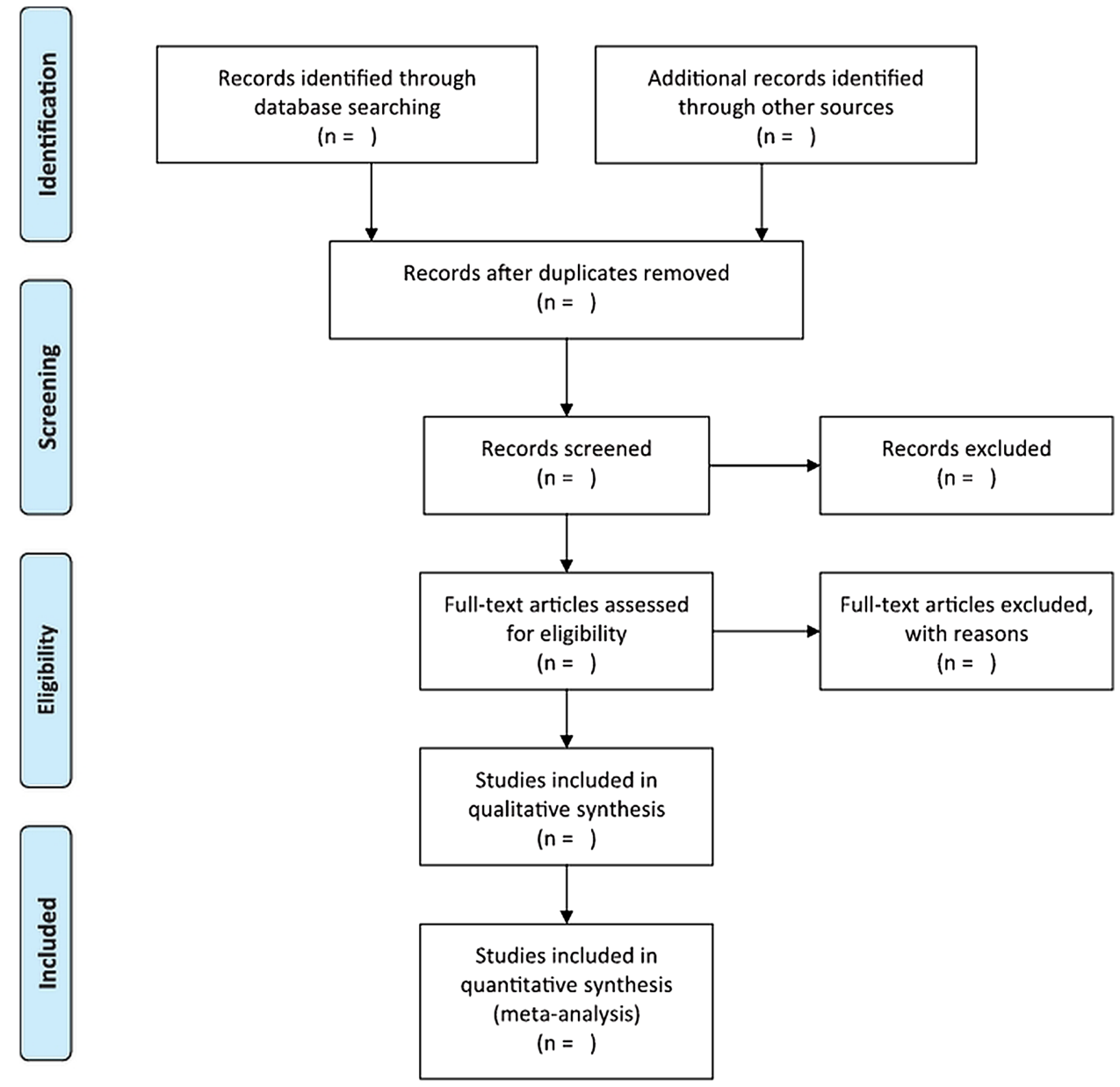

Figure 1 Study selection flow diagram.

\section{Assessment of heterogeneity}

We will use the random-effect or fixed-effect model for the meta-analysis according to the data analysis. If a meta-analysis is possible, we will use the $\mathrm{I}^{2}$ statistic to quantify the inconsistencies among the included studies. According to the guidance given in the Cochrane Handbook for Systematic Reviews of Interventions, 50\% will be the cut-off point for meaningful heterogeneity. If heterogeneity is observed, we will conduct a subgroup analysis to explore the possible causes. ${ }^{15}$

\section{Assessment of reporting biases}

If a sufficient number of included studies (at least 10 trials) are available, we will use funnel plots to detect reporting biases. ${ }^{16}$ However, funnel plot asymmetry is not the same as publication bias; therefore, we will attempt to distinguish the possible reasons for the asymmetry, such as small-study effects, poor methodological quality and true heterogeneity in the included studies. ${ }^{16} 17$

\section{Data synthesis}

The differences between the intervention and control groups will be assessed. The RR and 95\% CIs will be assessed for the effect size of each included study. All of the statistical analyses will be conducted using Cochrane
Collaboration's Software Program, Review Manager (RevMan), V.5.2.7 for Windows (Copenhagen, The Nordic Cochrane Centre, the Cochrane Collaboration 2012). For studies with insufficient information, we will contact the corresponding authors to acquire and verify the data when possible. $\chi^{2}$ and $\mathrm{I}^{2}$ tests will be used to evaluate the heterogeneity of the included studies. Unless excessive statistical heterogeneity is present, we will then pool the data across studies for a meta-analysis using a random-effects or fixed-effect model.

\section{Subgroup analysis and investigation of heterogeneity}

If the data are available, we will conduct subgroup analyses to assess the heterogeneity between the studies, including the following:

1. Type of BVA;

2. Type of control;

3. Duration of RA;

4. Laterality of RA, bilateral RA versus unilateral RA.

\section{Sensitivity analysis}

Sensitivity analysis will be conducted according to the following criteria: 
Sample size (small sample studies eg, less than 40 participants in each group, or large sample studies eg, more than 40 participants in each group);

1. Analysis-related issues (eg, processes to handle the missing data).

\section{DISCUSSION}

This systematic review will provide a detailed summary of the current state of evidence for the effects of BVA in treating symptoms in patients with RA. The review will benefit patients and practitioners in the fields of traditional and complementary medicine.

Contributors The protocol was drafted by all authors. It was revised and the final version approved by all authors.

Competing interests JAL, JC, K-JY, JHJ and MSL were supported by the Korea Institute of Oriental Medicine (K14400); MJS was supported by the same institute (K14380)

Provenance and peer review Not commissioned; externally peer reviewed.

Open Access This is an Open Access article distributed in accordance with the Creative Commons Attribution Non Commercial (CC BY-NC 3.0) license, which permits others to distribute, remix, adapt, build upon this work noncommercially, and license their derivative works on different terms, provided the original work is properly cited and the use is non-commercial. See: http:// creativecommons.org/licenses/by-nc/3.0/

\section{REFERENCES}

1. Han $\mathrm{A}$, Robinson $\mathrm{V}$, Judd $\mathrm{M}$, et al. Tai chi for treating rheumatoid arthritis. Cochrane Database Syst Rev 2004(3):CD004849.

2. Odegard S, Finset A, Kvien TK, et al. Work disability in rheumatoid arthritis is predicted by physical and psychological health status: a 7-year study from the Oslo RA register. Scand J Rheumatol 2005;34:441-7.

3. Yelin E. Work disability in rheumatic diseases. Curr Opin Rheumatol 2007;19:91-6.
4. Kvien TK, Uhlig T. Quality of life in rheumatoid arthritis. Scand J Rheumatol 2005;34:333-41.

5. Lubeck DP. Patient-reported outcomes and their role in the assessment of rheumatoid arthritis. Pharmacoeconomics 2004:22(2 Suppl 1):27-38

6. Munstedt K, Hackethal A, Schmidt K. Bee venom therapy, bee venom acupuncture of apipunture: what is the evidence behind the various health claims? Am Bee J 2005;145:665-68.

7. Lee MS, Pittler MH, Shin BC, et al. Bee venom acupuncture for musculoskeletal pain: a review. J Pain 2008;9:289-97.

8. Son DJ, Lee JW, Lee YH, et al. Therapeutic application of anti-arthritis, pain-releasing, and anti-cancer effects of bee venom and its constituent compounds. Pharmacol Ther 2007;115:246-70.

9. Baek YH, Huh JE, Lee JD, et al. Antinociceptive effect and the mechanism of bee venom acupuncture (Apipuncture) on inflammatory pain in the rat model of collagen-induced arthritis: mediation by alpha2-adrenoceptors. Brain Res 2006;10731074:305-10.

10. Chen HS, Qu F, He X, et al. The anti-nociceptive effect and the possible mechanism of acupoint stimulation caused by chemical irritants in the bee venom pain model. Brain Res 2010;1355:61-9.

11. Lee JD, Park HJ, Chae Y, et al. An overview of bee venom acupuncture in the treatment of arthritis. Evid Based Complement Alternat Med 2005;2:79-84.

12. Hauser RA, Daguio M, Wester D, et al. Bee-venom therapy for treating multiple Sclerosis: a clinical trial. Altern Complement Ther 2001;7:37-45.

13. Kwon YB, Kim JH, Yoon JH, et al. The analgesic efficacy of bee venom acupuncture for knee osteoarthritis: a comparative study with needle acupuncture. Am J Chin Med 2001;29:187-99.

14. Higgins JPT, Altman DG, Sterne JAC. Chapter 8: assessing risk of bias in included studies. In: Higgins JPT, Green S. eds. Cochrane handbook for systematic reviews of interventions version 510 (updated March 2011) The cochrane collaboration. http://www. cochrane-handbook.org, 2011.

15. Deeks JJ, Higgins JPT, Altman DG. Chapter 9: analysing data and undertaking meta-analyses. In: Higgins JPT, Green S. eds. Cochrane handbook for systematic reviews of interventions version 510 (updated March 2011) The cochrane collaboration. http://www. cochrane-handbook.org, 2011.

16. Sterne JAC, Egger M, Moher D. Chapter 10: addressing reporting biases. In: Higgins JPT, Green S. eds. Cochrane handbook for systematic reviews of interventions version 510 (updated March 2011) The cochrane collaboration. http://www.cochrane-handbook. org, 2011.

17. Egger M, Davey Smith G, Schneider M, et al. Bias in meta-analysis detected by a simple, graphical test. BMJ 1997;315:629-34. 